Meta

Journal des traducteurs

Translators' Journal

\title{
Théorie linguistique et théorie de la traduction
}

\section{Maurice Pergnier}

Volume 26, numéro 3, septembre 1981

URI : https://id.erudit.org/iderudit/003823ar

DOI : https://doi.org/10.7202/003823ar

Aller au sommaire du numéro

Éditeur(s)

Les Presses de l'Université de Montréal

ISSN

0026-0452 (imprimé)

1492-1421 (numérique)

Découvrir la revue

Citer cet article

Pergnier, M. (1981). Théorie linguistique et théorie de la traduction. Meta, 26(3), 255-262. https://doi.org/10.7202/003823ar d'utilisation que vous pouvez consulter en ligne.

https://apropos.erudit.org/fr/usagers/politique-dutilisation/ 


\section{Théorie linguistique et théorie de la traduction}

Maurice Pergnier

Je voudrais tout d'abord m'expliquer sur le titre donné à cet exposé. À un certain nombre de personnes, la distinction faite entre théorie linguistique et théorie de la traduction apparaîtra en effet sans objet, car pour elles la traduction fait partie intégrante de la linguistique et - ajouteraient-elles - c'est une des branches de la linguistique appliquée. Il leur paraitrait donc plus normal de parler de «théories linguistiques appliquées à la traduction». Pour d'autres, moins nombreuses, la théorie de la traduction n'a guère de rapports avec la linguistique, et elles seraient tentées de ne voir dans ce titre qu'une juxtaposition de deux domaines de préoccupation entièrement séparés. Enfin chacun peut observer par lui-même qu'il n'existe pas une théorie linguistique et une théorie de la traduction, mais une multiplicité de théories linguistiques et une multiplicité de théories de la traduction. En intitulant cet exposé «Théorie linguistique et théorie de la traduction», j'ai donc voulu marquer volontairement que, selon moi, la traduction n'est pas une annexe de la linguistique, mais qu'elle relève au contraire d'un cadre théorique spécifique. C'est un premier point sur lequel je devrai $m$ 'expliquer ici, et je le ferai dans une première partie.

Je m'empresse cependant d'ajouter que le fait de dire que la théorie linguistique et la théorie de la traduction ne se confondent pas, ou que l'une n'est pas l'application pure et simple de l'autre, n'implique évidemment pas que ce soit là deux disciplines distinctes, et que la théorie de la traduction n'ait rien à emprunter aux théories linguistiques, ni que les théories linguistiques ne puissent pas s'enrichir des apports de la recherche en traduction.

C'est au contraire l'inverse que je voudrais montrer. Théorie de la traduction et théorie linguistique sont en effet totalement imbriquées et solidaires l'une de l'autre, puisque l'objet de la linguistique est le langage et que la traduction est l'une des manifestations de langage parmi d'autres. Ce que je veux souligner ici et que j'essaierai de justifier dans ma deuxième partie, c'est que la théorie de la traduction n'a de chance de progresser, dans le cadre de théories plus vastes du langage, que si elle fait l'objet d'une recherche spécifique partant de ses propres préoccupations, et ne se contente pas de vouloir plaquer sur la traduction des modèles tout faits et qui n'ont pas été élaborés pour elle.

J'ajoute que non seulement c'est la seule façon pour la théorie de la traduction de progresser en mettant en accord les modèles théoriques et la pratique, mais aussi que la linguistique générale elle-même a tout à y gagner, car la traduction est un phénomène central dans le fonctionnement et l'étude de langage, et il y a fort à parier que lorsque ses mécanismes fondamentaux auront 
été explicités c'est l'ensemble de notre connaissance des mécanismes présidant au fonctionnement de la parole et à la structuration des langues qui aura fait un pas important.

La linguistique est une discipline qui se veut scientifique mais c'est une science qui manque singulièrement de procédures de vérifications expérimentales. Or, il n'est guère de discipline qui puisse se dire scientifique si elle ne procède pas par un mouvement d'aller-et-retour constant entre l'établissement de modèles théoriques et les vérifications expérimentales. Dans le domaine du langage comme ailleurs, l'absence de procédures de vérification ouvre la porte à la théorisation gratuite. Parmi les champs de vérification expérimentale qui s'ouvrent aux modèles linguistiques, il n'en est peut-être que deux. L'un est la pathologie du langage - notamment l'étude des aphasies, et l'autre est la traduction. C'est pourquoi je place pour ma part les problèmes soulevés par la traduction au centre de toute théorie linguistique. J'y reviendrai après m'être penché tout d'abord sur les deux premiers points soulevés.

Je voudrais donc parler tout d'abord des raisons pour lesquelles la théorie de la traduction me semble devoir être élaborée de manière autonome par rapport à l'ensemble des théories linguistiques. Ces raisons sont de trois ordres différents mais complémentaires.

Premièrement, la traduction n'est pas un fait statistique, un fait «de structures », mais au contraire un fait dynamique. Les linguistes ont quelquefois été enclins à confondre la traduction avec la linguistique différentielle, discipline comparative qui s'intéresse aux faits de langue hors message pour approfondir notre connaissance des différents systèmes linguistiques qui peuvent se trouver en interférence soit à travers la traduction, soit pour toute autre raison. Or, parce qu'elle est un processus dynamique de recherche d'équivalences entre des messages, la traduction met en jeu non seulement des systèmes linguistiques mais aussi des processus psychologiques de compréhension et d'expression, ainsi que des processus de mobilisation de connaissances extra-linguistiques qui ont été bien décrits par D. Seleskovitch et également par J. Delisle. Elle relève donc autant de la psychologie et de la psycholinguistique que de la linguistique. Elle met d'autre part en jeu des phénomènes de culture et de civilisation qui la font tomber, à travers la sociolinguistique, dans le champ de la sociologie.

En second lieu, la théorisation sur la traduction, comme toute théorisation sur une pratique, a tout intérêt à s'appuyer sur une expérience pratique ou du moins sur une observation de première main. Or tous les linguistes ne sont pas traducteurs et ne sont pas nécessairement conscients des caractères spécifiques de cette activité. Ce sont les praticiens de la traduction qui sont les plus aptes, sinon à résoudre, du moins à poser correctement les problèmes théoriques, et à les formuler dans des termes où des réponses peuvent leur être apportées. De ce point de vue, le souci tout récent de traducteurs professionnels de théoriser eux-mêmes leur art est un apport de toute première importance. Jusqu'à récemment, en effet, la théorisation sur la traduction était laissée aux seuls linguistes et psychologues, avec le résultat que la théorie se perdait assez souvent dans les sables de faux problèmes mal posés. Qu'on pense par exemple aux débats nébuleusement philosophiques qu'ont fait naître les théories de 
Whorf-Sapir, sur les «visions du monde» et dont la conclusion logique devait être inéluctablement que la traduction est impossible. Au lieu de s'interroger pour savoir sur quoi et comment la traduction opérait, on la déclarait impossible ou traîtresse en projetant sur elle le modèle théorique d'une sémantique structurale réductionniste qui assimilait catégories grammaticales et catégories conceptuelles. Whorf-Sapir et leurs disciples américains ou européens n'étaient pas de mauvais linguistes. Ils extrapolaient seulement d'un domaine de la linguistique sur un autre et prenaient la partie pour le tout, c'est-à-dire en l'occurrence les systèmes linguistiques pour le sens et la comparaison de systèmes pour l'opération traduisante. Il est certain que la recherche théorique sur la traduction, menée par des praticiens de la traduction qui ne posent pas comme première question : pourquoi? mais : comment?, est de nature à éviter grandement ce genre de problème.

La troisième raison tient aux conditions mêmes dans lesquelles la linguistique s'est développée et à la façon dont elle a ordonné sa propre problématique. Un reproche qui est souvent adressé à la linguistique par les traducteurs qui cherchent chez les linguistes des réponses aux questions qu'ils se posent concernant leur activité, est que la linguistique s'intéresse uniquement au fonctionnement et à la structure des langues, et non à la parole réelle. C'est un reproche qui n'est que partiellement justifié, ainsi que j'essaierai de le montrer tout à l'heure. Mais il est vrai, dans l'ensemble, que l'axe du développement de la linguistique des cinquante dernières années s'est situé beaucoup plus du côté des structures de la langue que du côté des mécanismes de la parole. L'un des défauts qu'on peut même reprocher à de nombreuses écoles linguistiques qu'elles soient d'inspiration saussurienne, en Europe, ou bloomfeldiennes, en Amérique du Nord - a été de vouloir couper la langue de la parole, c'est-à-dire de la réalité vivante du langage. Ce défaut a atteint son point culminant et de manière presque caricaturale avec la linguistique de Chomsky, mais il est plus ou moins imputable à toutes les écoles linguistiques jusqu'aux années 70 . Certes les justifications épistémologiques ne manquent pas pour appuyer ce choix de l'étude prioritaire des structures linguistiques au détriment de l'étude de la parole. Il serait naif de croire, par exemple, que Saussure ignorait que la parole était aussi importante à l'étude globale du langage que la langue. Saussure annonce très clairement que la linguistique comporte deux branches complémentaires : la linguistique de la langue et la linguistique de la parole. Si les successeurs de Saussure et de Bloomfield ont choisi de développer en priorité l'étude de la langue, c'est-à-dire des structures sous-jacentes à l'exercice de la parole et à la communication par le langage, c'est qu'ils étaient conscients de ce que seule celle-ci constituait un terrain solide pour faire progresser la connaissance scientifique du langage en introduisant un ordre dans ce qui autrement resterait une globalité inanalysable. On ne peut que constater après coup que leur choix était juste. Sans la décision épistémologique prise par les «pères fondateurs » de la linguistique de s'attaquer tout d'abord à la mise à jour des structures et à l'élucidation des rapports existant entre ces structures et le contenu de la parole, notre connaissance du langage serait aussi confuse et aussi chaotique qu'il y a un siècle. Bien qu'il existe encore de très nombreuses doctrines linguistiques différentes, on peut dire que la linguistique s'est fondée à 
ce jour un corps de méthodes et de connaissances faisant l'objet d'un large consensus, ce qui est la garantie de leur scientificité. À partir de ce noyau dur, elle est donc en mesure d'aborder avec rigueur l'étude des rapports entre la langue et la parole.

Il reste que, pendant un demi-siècle, la linguistique de la parole est restée très en arrière de la linguistique de la langue. Or, c'est surtout dans une théorie de la parole que la théorie de la traduction aurait besoin de puiser. La traduction, en effet, tout le monde commence à en convenir, chez les linguistes comme chez les traducteurs, est une opération linguistique portant sur des contenus de sens propres à des messages particuliers et non une recherche d'équivalences entre des unités appartenant à deux langues en tant que telles. C'est donc, en termes saussuriens, un acte de parole. Le développement encore insuffisant de l'étude de la parole chez les linguistes doit amener - et amène effectivement - les théoriciens de la traduction à élaborer eux-mêmes, à partir de l'étude de la traduction, des modèles de fonctionnement de la parole. C'est en effet dans leur domaine d'application que le besoin s'en fait sentir de la manière la plus urgente. Il est certain aussi que la traduction est un terrain privilégié pour l'élaboration de modèles explicatifs en ce domaine et que l'ensemble de la linguistique bénéficie des apports de recherches comme celles effectuées par D. Seleskovitch et son école sur les mécanismes de la compréhension et de la reformulation dans l'interprétation consécutive et simultanée, car l'opération traduisante met en lumière des phénomènes qui, sans ce révélateur, resteraient enfermés dans la «boîte noire » du psychisme humain.

L'intérêt des linguistes pour la traduction est relativement récent. Il serait cependant très injuste de tenir pour négligeables les contributions faites par des linguistes à la théorie de la traduction dès les années 60 . On ne citera ici que les plus importantes qui sont la «Stylistique comparée du français et de l'anglais» de Vinay et Darbelnet» et «Toward a Science of Translating» d'E. Nida, deux ouvrages qui ouvraient, chacun à sa façon, les voies nouvelles dans lesquelles la théorie de la traduction allait s'engager. E. Nida, pour la première fois, tentait de construire une théorie de la traduction en s'appuyant sur des instruments d'analyse rigoureux issus à la fois des différentes théories linguistiques de la langue et de sciences voisines comme la théorie de l'information, c'est-à-dire en tentant de jeter le pont entre la langue et la parole. Quant à l'ouvrage de Vinay et Darbelnet, il constituait une étape considérable dans le développement d'une linguistique orientée vers la traduction. Cela est si vrai que, du moins chez les francophones, toutes les études sur la traduction qui se sont développées récemment se sont élaborées, soit en complément de cette méthode d'approche, soit en réaction contre elle, preuve qu'elle avait touché quelque chose d'essentiel dans les fondements de l'opération traduisante. Son mérite essentiel était d'avoir dégagé la notion même de traduction de ses connotations de transposition servile de mots d'une langue en mots d'une autre langue et d'avoir montré à l'aide d'une multitude d'exemples et d'un corps systématique de procédures de passage d'une langue à l'autre, que la traduction pouvait à la fois être fidèle au sens et ne pas massacrer la langue d'arrivée en y injectant des calques approximatifs de la langue de départ. De ce point de vue, la stylis- 
tique comparée était en même temps un ensemble cohérent et structuré de techniques de traduction, et un manifeste en faveur d'une traduction de qualité contre le médiocre transcodage qui souvent en tient lieu et qui est une offense aux langues aussi bien qu'aux informations véhiculées par la traduction. De ce point de vue, les mérites de ce livre restent intacts. Sa faiblesse, du point de vue de l'évolution ultérieure de la pensée sur la traduction, tient à ce que - bien qu'il ait à de nombreux égards devancé la pensée linguistique de son époque - il reste encore très prisonnier de la linguistique de la langue, et traite de la traduction comme d'une opération se situant uniquement au niveau du syntagme, sans marquer suffisamment le fait que la traduction d'un terme ou d'une structure nécessite le passage par une phase d'analyse qui ne tient pas à la comparaison des structures lexicales et grammaticales de deux langues en présence. Les notions de message et de sens en situation n'en sont certes pas absentes, mais elles restent à l'arrière-plan, comme en filigrane, les auteurs n'ayant pas en tête de démontrer les mécanismes de l'opération traduisante, mais de montrer comment il est possible - entre deux langues comme le français et l'anglais - de trouver des équivalents en contexte à toutes les structures (lexicales, grammaticales ou stylistiques) en évitant les contaminations de la littéralité.

Le fait que des recherches ultérieures - comme c'est le cas notamment pour les miennes - aient déplacé le centre de gravité en direction des équivalences de mots en situation - c'est-à-dire dans les textes - n'empêche pas les considérations différentielles de la $\mathrm{S}$.C. d'être toujours valables au niveau où elles se placent et intégrables à une théorie globale de la traduction.

A cet égard, il est à noter que les lignes de développement de la recherche linguistique et de la recherche en traduction vont plus souvent suivre des cours parallèles que s'opposer. Cela était vrai lorsque régnait la linguistique structurale ou fonctionnelle, et où la réflexion sur la traduction était essentiellement différentielle au niveau des structures linguistiques, et insistait - je pense notamment aux travaux de Mounin - sur l'intraduisibilité, c'est-à-dire sur l'intransposabilité des structures. Cela est encore vrai aujourd'hui, puisqu'on constate que des théories de la traduction fondées sur l'analyse du discours et sur le sens en situation - comme celle de D. Seleskovitch - sont contemporaines de l'importance prise dans la linguistique (et indépendamment de la traduction) par des courants de pensée comme la «pragmatique», ou la «speech act theory» de Austin et Searle, qui insistent, au contraire des écoles précédentes, sur tous les éléments extérieurs à la systématique de la langue.

À cet égard, il n'est pas sans intérêt de constater que l'avancée la plus audacieuse dans cette direction n'est pas venue de la linguistique, mais de D. Seleskovitch, partant d'une théorie qu'elle veut volontiers polémique vis-àvis de la linguistique, preuve que la recherche sur la traduction peut progresser à partir de n'importe quel aspect de cette activité, et qu'il ne faut pas que les chercheurs en traduction attendent des modèles linguistiques adéquats pour élaborer des modèles théoriques de la traduction.

Il serait cependant dommage qu'à une linguistique uniquement centrée sur la langue succèdent des études uniquement centrées sur la parole, qu'après le 
règne presque exclusif du signifié s'instaure le règne exclusif du «sens». On tomberait alors dans un excès inverse mais tout aussi injustifié. Car, qu'on le veuille ou non, dans la parole normale comme dans la traduction, le sens - si transcendant soit-il aux composantes linguistiques - est toujours formalisé et véhiculé par des signes linguistiques. Il n'y a, par exemple, pas de texte traduit qui ne parte d'un sens émis dans une langue, et qui n'aboutisse à un texte émis dans une autre langue. De même, tout fait de langage est le résultat d'une dialectique entre une forme linguistique et un contenu de sens. Le nier serait penser que je pourrais aujourd'hui m'exprimer en japonais sans jamais l'avoir appris, pourvu que j'aie quelque chose d'intéressant à dire.

Mais les convergences et les complémentarités entre toutes ces approches sont tout à fait remarquables, et on peut dire qu'on est aujourd'hui bien plus prêt qu'on ne l'a jamais été d'une véritable théorie de la traduction. Dire que la traduction est un fait de parole et recentrer son étude autour de l'étude de la compréhension et de l'expression des sens par-delà des mots - comme je l'ai fait moi-même dans mon travail sur les fondements socio-linguistiques de la traduction - ce n'est donc pas rejeter comme inutile la contribution de la linguistique de la langue. C'est redonner à chacune sa juste place. De même qu'il n'y a pas de langue sans parole, il n'y a pas de parole sans langue. Vouloir tout ramener à l'une sans tenir compte de l'autre, c'est à notre avis vouloir faire une tapisserie en utilisant seulement la trame ou seulement la chaîne. Le fait qu'une opération de traduction porte sur du sens c'est-à-dire sur le contenu d'un message n'empêche pas que ce sens soit appréhendé à travers des signifiés et que ce soit à nouveau des signifiés qui réexprimeront ce sens dans la langue de la traduction. Ces signifiés nous renvoient donc inéluctablement au système linguistique.

Il est certes intéressant de constater que les différentes études théoriques sur la traduction qui se sont faites jour ces dernières années, venant de la linguistique - comme celles de Nida ou la mienne, par exemple - et celles venant de la «traductologie», - comme celle de D. Seleskovitch - convergent sur un nombre important de points fondamentaux qu'on peut formuler schématiquement de la façon suivante:

- La traduction recherche non des équivalences entre des signes linguistiques en tant que tels, mais entre des signes linguistiques en situation.

- Cette recherche d'équivalences suppose que les différents composants de l'énoncé traduit soient référés non seulement au contexte immédiat, mais à l'ensemble des connaissances extra-linguistiques qui conditionnent l'énonciation et la compréhension du message. (C'est ce que D. Seleskovitch appelle les «compléments cognitifs» et ce que j'appelle pour ma part «les paramètres situationnels du message »).

Il est à noter que ces notions étaient déjà présentes dans des théories linguistiques de la traduction comme celle de Vinay et Darbelnet ou celle de Nida première manière, mais seulement en arrière-plan, comme un ensemble d'intuitions non explicitées et non théorisées. Leur mise à jour et leur intégration à un corps de doctrine organisé manifeste en même temps une avancée dans le domaine de la linguistique théorique et une avancée importante dans la prise de 
conscience de la spécificité de la traduction, spécificité trop souvent niée ou ignorée par tous ceux qui croient que la traduction repose sur un simple transcodage des signes composant les énoncés.

Si la traduction ne peut pas opérer selon ce principe simpliste c'est que les langues sont des réalités beaucoup plus complexes aussi que ne le croient la majorité des gens. Et la linguistique se doit d'expliquer pourquoi la transposition pure et simple des mots n'aboutit pas à une traduction digne de ce nom.

C'est dire que l'avenir semble appartenir - pour la théorie de la traduction comme pour la linguistique générale - à des recherches qui tiendraient les deux bouts de la chaîne, c'est-à-dire aussi bien l'étude du système linguistique (et des systèmes linguistiques en interférence), que l'étude de leur actualisation dans la parole. Car, répétons-le, il n'y a pas de parole sans système linguistique, et pas de système linguistique sans parole. La constitution et le fonctionnement de la parole sont incompréhensibles sans la référence à la nature du système.

Hélas! l'étude du langage, comme tous les domaines où la théorisation tient plus de place que la vérification expérimentale, est soumise à des phénomènes de mode et à des fluctuations qui la font progresser en zig-zag, en jetant souvent par-dessus les moulins les acquis des écoles précédentes. Il n'est donc pas rare d'y voir prendre la partie pour le tout, en changeant périodiquement le centre d'intérêt, décrété, pour les besoins de la cause, être le centre autour duquel s'organise la totalité de la théorie linguistique. C'est ainsi qu'après la vogue exclusive de la structure paradigmatique, on a assisté à la vogue exclusive de l'ambiguïté syntaxique et de la transformation, avant d'assister maintenant à la vogue montante de la "présupposition", selon laquelle tout s'expliquerait dans le langage par les différents présupposés inscrits dans chaque forme linguistique. S'il n'est pas niable que ces différents apports théoriques contribuent de manière intéressante au progrès de la connaissance du langage, on peut contester leur prétention à être chacune «une » théorie du langage qui en remplace ou en supplante d'autres. Il n'est pas douteux, au contraire, que toutes doivent pouvoir s'intégrer à une théorie plus vaste dont elles constitueraient un élément pour pouvoir prétendre au statut de modèles théoriques explicatifs. Faute de quoi, elles restent seulement un ensemble d'observations coordonnées sur la base d'une certaine hypothèse théorique.

Puisque nous parlons ici des théories linguistiques dérivées des travaux d'Austin, théories qui rejoignent incontestablement les préoccupations des théoriciens de la traduction, soulignons une de leurs insuffisances qui tient à cette tendance que nous dénoncions, de prendre la partie pour le tout. Cette insuffisance est la suivante: les auteurs ne nous disent jamais, quand il est question de présupposés, si ces présupposés sont de langue (c'est-à-dire inscrits dans la sémantique et la syntaxe de la langue) ou s'ils sont de parole (c'est-à-dire inscrits dans la situation d'émission et de réception des messages.) Or, cette distinction est de toute première importance pour le théoricien - comme pour le praticien - de la traduction, qui a le plus grand besoin de distinguer entre le vouloir-dire des signifiés linguistiques et les apports de sens consécutifs à leur insertion dans des textes. Il est indéniable que si ces travaux s'inscrivaient dans 
les acquis préalables de la sémantique et de la linguistique générale, au lieu de se concevoir de manière autonome, leur apport pour l'ensemble de la théorie linguistique pourrait être plus riche.

C'est là aussi un exemple de domaine où la théorie de la traduction et la théorie sémantique pourraient s'apporter une contribution mutuelle. Car il est évident que le traducteur confronté avec les «présupposés» d'un texte ne les traitera pas de la même façon selon qu'ils sont de langue ou de parole. Il pourra donc apporter, s'il se penche sur des notions de ce genre, en testant leur applicabilité à la traduction, une utile contribution à la théorie sémantique. Mais s'il opère une coupure trop radicale entre ce qu'il appellera - avec D. Seleskovitch - la «signification en discours», et la «signification en langue» supposant l'une sans rapport avec les autres, comme si ces deux types de «signification» habitaient deux planètes différentes, comment pourra-t-il expliquer que les mots puissent être de quelque façon compris et traduits?

En conclusion, il nous paraît tout à fait illusoire de penser, soit que la théorie linguistique de la langue - et notamment la sémantique - puisse progresser indépendamment de l'étude de la façon dont les signifiés s'actualisent dans la parole vivante, car la langue n'est pas une entité close sur elle-même mais au contraire une réalité qui n'existe que par et pour la parole. Inversement il nous paraît tout aussi illusoire de penser que l'étude de la parole - et, en conséquence, celle de la traduction - puisse se passer de théories de la langue, ou plus précisément d'une théorie concernant les rapports qu'entretiennent la langue et la parole.

Tant qu'elle est descriptive - c'est-à-dire tant qu'elle prend acte simplement des opérations qui s'enchaînent dans la traduction - la théorie de la traduction peut se passer d'une théorie de la langue; mais dès qu'elle veut devenir explicative - par exemple si elle veut justifier pourquoi la traduction par transcodage n'aboutit pas vraiment à une traduction - la théorie de la traduction est obligée de faire appel à des concepts qui relèvent de la théorie de la langue.

Je crois donc, pour ma part, que théorie de la traduction et linguistique générale sont condamnées à travailler et à progresser ensemble, non seulement parce que la linguistique - ou plutôt, certaines branches de la linguistique apportent à la théorisation de la traduction des concepts et des instruments d'analyses qui sont indispensables à l'examen d'un phénomène aussi complexe que la traduction, mais aussi parce qu'une théorisation de la traduction à partir d'une expérience de sa pratique est de nature à faire progresser, par-delà la théorie de la traduction elle-même, tous les aspects de la théorie linguistique, y compris la linguistique de la langue. 